\title{
AVANT-PROPOS AU SECOND VOLUME
}

Mon ami, Norman Hampson, de l'Université de Manchester, a bien voulu revoir les premières épreuves des Livres II et III qui forment ce volume. Les observations dont il m'a fait part et les conseils qu'il m'a prodigués, à la suite d'une lecture minutieuse, m'ont permis à la fois d'atténuer certaines affirmations trop sommaires et de profiter de ses appréciations sur la politique gouvernementale afin de mieux placer dans la conjoncture du tournant de 1793-1794 une étude consacrée surtout à l'action anarchique de ceux qui, n'étant pas hommes de gouvernement, faisaient peu de cas des considérations de la haute politique. Ses remarques au sujet du Livre Trois m'ont été particulièrement profitables et je lui suis très reconnaissant d'une aide en même temps si critique, si avisée et si bienveillante. Qu'il trouve ici l'expression émue de mes remerciements.

$M^{\text {Ile }}$ Margaret Tennant, ancienne étudiante à l'Université de Leeds, a entrepris la tâche ingrate de fournir à cette étude consacrée à des hommes modestes et à des institutions éphémères l'appareil indispensable d'un index facilement consultable qui servira au lecteur de boussole à travers un livre qui est aussi voyageur que cette Terreur ambulante de l'an II. A elle je dois une grande dette de reconnaissance, non seulement pour le soin et l'intelligence qu'elle a mis à accomplir un travail fastidieux, mais encore pour l'encouragement amical qu'elle m'a fourni dans cette dernière étape.

La personne qui m'a le plus aidé dans ma vie de chercheur n'est malheureusement plus ici pour voir paraître ce volume.

Leeds, juillet 1962. 